LETTER TO THE EDITOR

\title{
Validating a gene expression signature proposed to differentiate liposarcomas that use different telomere maintenance mechanisms
}

Oncogene (2012) 31, 265-266; doi:10.1038/onc.2011.225; published online 27 June 2011

A gene expression signature meant to distinguish between telomerase and alternative lengthening of telomeres (ALT) utilization was recently described (Lafferty-Whyte et al., 2009). This was generated from the intersection of two independent signatures obtained from cell lines and liposarcoma tumors, respectively, which utilize different telomere maintenance mechanisms (TMMs). To assess the utility of this signature, we used U133plus2.0 arrays (Affymetrix, Santa Clara, CA, USA) to undertake a similar analysis of an independent collection of liposarcomas with defined TMMs (see GEO accession GSE20559, which includes full details of the methods). For our data set, use of the 297 gene signature causes the liposarcomas to cluster not on the basis of TMM, but rather on the basis of tumor histological subtype (Figure 1a), consistent with the signatures reported by others (Matushansky et al., 2008).

To control for the effect of histological subtypes, we then restricted our analysis to dedifferentiated liposarcomas. Despite this constraint, when using linear modeling for microarrays (Limma; (Smyth, 2004)) to assess differential expression, we obtained no genes that are significantly changed, unless the data are pre-filtered to remove low-expressing non-varying probesets $(\mathrm{cv}<0.1$ across the samples). The pre-filtered data generated a 14-gene signature (threshold of false discovery ratecorrected $P$-value $<0.1$, fold-change $>2$ ), with no genes in common with the 297-gene signature of Lafferty-Whyte et al. (2009). This is in agreement with the previous

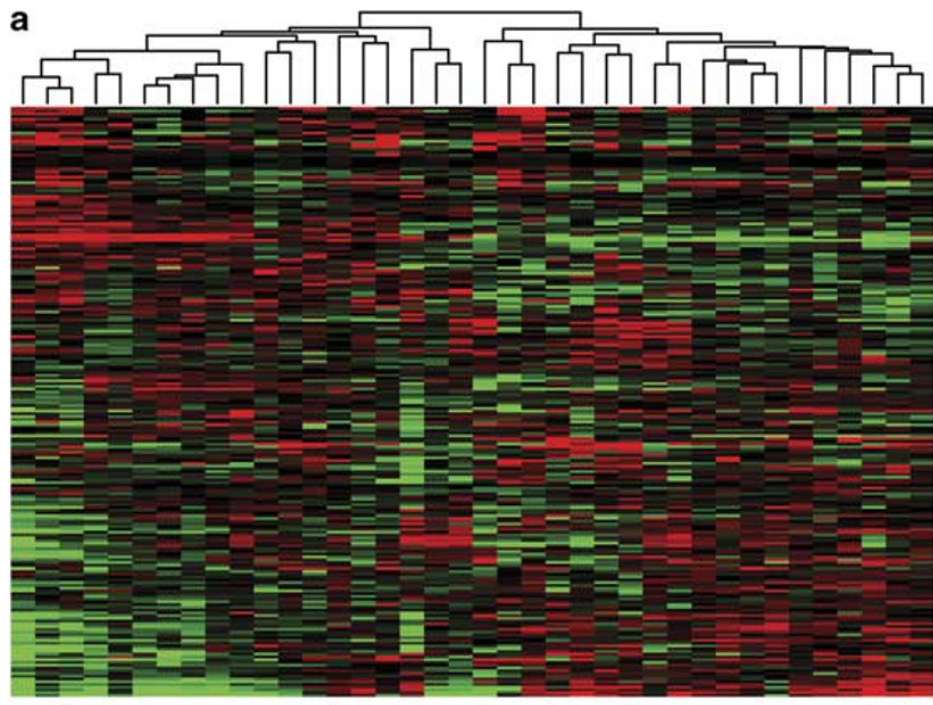

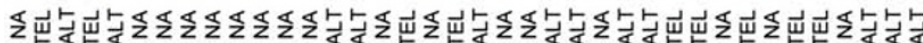

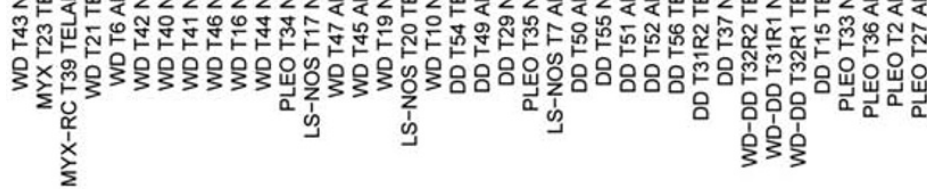

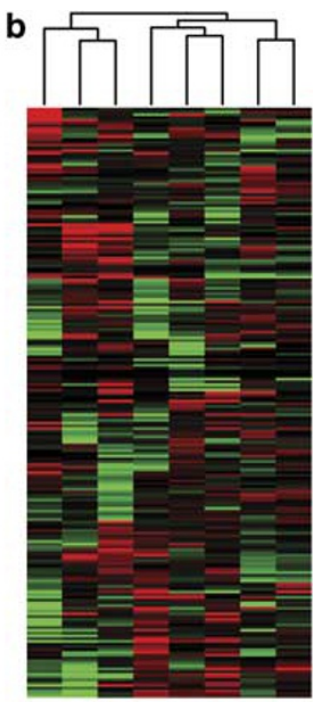

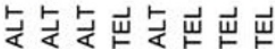

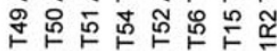

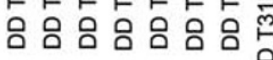

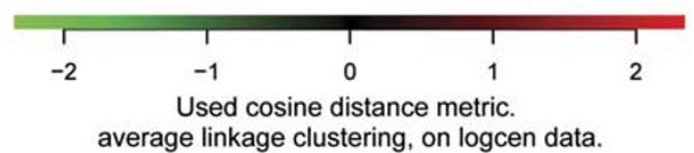

Figure 1 Heatmap visualizations of the 297 gene signature from Lafferty-Whyte et al. (2009) for all genes having a corresponding Affymetrix probeset. Where more than one probeset was available, the one with the highest median expression across the dataset was used as the exemplar. The data are GCRMA normalized and represented as cosine similarity on median-centered log-space data, using average linkage clustering. Red and green correspond to increased versus decreased expression, respectively, as compared with the experiment-wide median. Histological type (WD, well differentiated; MYX, myxoid; RC, round cell; DD, dedifferentiated; PLEO, pleomorphic; LS NOS, liposarcoma not otherwise specified) and TMM are indicated, with 'NA' used for tumors that were not clearly ALT or telomerase-positive. (a) Heatmap of 38 tumors representing all histological subtypes of liposarcoma. (b) Heatmap of only dedifferentiated liposarcoma samples. 
study, where a similar approach failed to identify any genes that are significantly correlated with TMM, an observation that motivated their use of Fisher's exact test to produce a list of $>6700$ differentially expressed genes. We also performed unsupervised clustering of the dedifferentiated tumor data corresponding to the 297-gene signature (Figure 1b) and found no strong correlation between gene expression and TMM.

It is possible that the discrepancies between our data and those of Lafferty-Whyte et al. (2009) reflect differences in microarray platform or tumor samples used. However, as the authors noted, the cell lines used in their study differ not only in TMM, but also in tissue of origin, with $75 \%$ of the telomerase-positive cell lines being of epithelial origin and $75 \%$ of the ALT cell lines being of fibroblastic origin. The initial gene signature obtained from comparing these two groups would be expected to contain a strong signature reflective of cell type, in addition to any putative TMM signature. Likewise, although we note that the histological subtypes of liposarcomas used to generate the 297 gene signature were not specified in this publication, or in the information uploaded into the GEO database, it can be largely deduced from an earlier publication from the same group (Cairney et al., 2008). Based on Table 1 in that report, we note that the ALT tumors are predominantly dedifferentiated liposarcomas, whereas the telomerase tumors are predominantly myxoid/round cell liposarcomas. Thus, a comparison of ALT versus telomerase tumors using this data set suffers from the confounding effect of differing histological subtypes in the two groups. Given the large-scale gene expression changes seen between cells of different origin and tumors of different histologies, it is perhaps not surprising that the 297 gene signature contains a strong component related to differentiation state, as shown in Figure 1a.

Dedifferentiated liposarcomas differ markedly from myxoid liposarcomas; different genetic mechanisms drive the formation of these two types of liposarcoma. Dedifferentiated liposarcomas harbor complex karyotypes commonly containing Chr.12q12-15 amplification, whereas $90 \%$ of myxoid/round cell liposarcomas are characterized by a $\mathrm{t}(12 ; 16)(\mathrm{q} 13-14 ; \mathrm{p} 11)$ translocation. Indeed, translocation driven sarcomas are generally telomerase positive, whereas those with complex karyotypes utilize ALT at an appreciable frequency (Montgomery et al., 2004; Ulaner et al., 2004), underscoring the bias in the sample set analyzed. This likely introduces a major confounding effect in their data set, with the set of ALT tumors heavily biased for dedifferentiated liposarcomas. As the expression signature of dedifferentiated liposarcoma more closely resembles mesenchymal stem cells than does that of myxoid/round cell liposarcoma (Matushansky et al., 2008), this may provide an explanation for their finding that ALT tumors appear to cluster with the mesenchymal stem cell samples. Certainly, our observation that the 297 gene signature fails to distinguish tumors based upon TMM in our independent set of tumors comprising a single histological type of liposarcoma suggests that this signature is not a robust indicator of TMM. Instead, it is likely that this 297-gene signature reflects differentiation differences associated with varying histological subtypes of liposarcoma.

\section{Conflict of interest}

The authors declare no conflicts of interest.

\section{Acknowledgements}

This work was supported by a research grant from The Liddy Shriver Sarcoma Initiative.

KR Doyle ${ }^{1}$, MA Mitchell ${ }^{1}$, CL Roberts ${ }^{1}$, S James $^{1}$, JE Johnson ${ }^{2}$, Y Zhou ${ }^{3}$, M von Mehren ${ }^{4}$, D Lev ${ }^{5}$, D Kipling ${ }^{6}$ and D Broccoli ${ }^{1}$ ${ }^{1}$ Department of Laboratory Oncology Research, Curtis and Elizabeth Anderson Cancer Institute, Memorial University Medical Center, Savannah, GA, USA,

${ }^{2}$ Department of Pathology and Laboratory Medicine, School of Medicine, University of Pennsylvania, Philadelphia, PA, USA,

${ }^{3}$ Bioinformatics Facility, Fox Chase Cancer Center, Philadelphia, PA, USA;

${ }^{4}$ Department of Medical Oncology, Population Science Division and Human Genetics Program, Fox Chase Cancer Center, Philadelphia, PA, USA; ${ }^{5}$ Department of Cancer Biology, The University of Texas MD Anderson Cancer Center, Houston, TX, USA and ${ }^{6}$ Department of Pathology, School of Medicine, Cardiff University, Cardiff, UK E-mail:broccdo1@memorialhealth.com

\section{References}

Cairney CJ, Hoare SF, Daidone MG, Zaffaroni N, Keith WN. (2008). High level of telomerase RNA gene expression is associated with chromatin modification, the ALT phenotype and poor prognosis in liposarcoma. Br J Cancer 98: 1467-1474.

Lafferty-Whyte K, Cairney CJ, Will MB, Serakinci N, Daidone MG, Zaffaroni $\mathrm{N}$ et al. (2009). A gene expression signature classifying telomerase and ALT immortalization reveals an hTERT regulatory network and suggests a mesenchymal stem cell origin for ALT. Oncogene 28: 3765-3774.

Matushansky I, Hernando E, Socci ND, Matos T, Mills J, Edgar MA et al. (2008). A developmental model of sarcomagenesis defines a differentiation-based classification for liposarcomas. Am J Pathol 172: 1069-1080.

Montgomery E, Argani P, Hicks JL, DeMarzo AM, Meeker AK. (2004). Telomere lengths of translocation-associated and nontranslocationassociated sarcomas differ dramatically. Am J Pathol 164: 1523-1529.

Smyth GK. (2004). Linear models and empirical bayes methods for assessing differential expression in microarray experiments. Stat Appl Genet Mol Biol 3: Article3.

Ulaner GA, Hoffman AR, Otero J, Huang HY, Zhao Z, Mazumdar M et al. (2004). Divergent patterns of telomere maintenance mechanisms among human sarcomas: sharply contrasting prevalence of the alternative lengthening of telomeres mechanism in Ewing's sarcomas and osteosarcomas. Genes Chromosomes Cancer 41: 155-162.

\footnotetext{
(c) (1)(2) This work is licensed under a Creative Commons Attribution-NonCommercial-ShareAlike 3.0 Unported License. To view a copy of this license, visit http:// creativecommons.org/licenses/by-nc-sa/3.0/
} 